\title{
VISIÓN ARTIFICIAL PARA RECONOCIMIENTO DE MANGOS EXPORTABLES UTILIZANDO REDES NEURONALES
}

\author{
Hugo Froilán Vega Huerta
}

\section{Resumen}

En este artículo explicaremos como se reconoce los mangos exportables basándonos únicamente en su forma. El proceso consiste en que, primeramente se selecciona un grupo de mangos exportables que cumplen con las exigencias de calidad, luego, mediante una cámara digital se captura la imagen de cada mango y mediante un software deprocesamiento de imágenes, cada mango será asociado a una imagen que contenga solamente la figura del borde del mango. Con dichas imágenes se procede con elentrenamiento de redes neuronales hasta encontrar una que sea capaz de reconocer acertadamente todos los elementos de la muestra. Luego, se procede con las pruebas de reconocimiento utilizando nuevos mangos que pueden poseero no un tamaño ideal. Si la red neuronal entrenada reconoce adecuadamente el tamaño de dicho mango,podremos decir que hemos cumplido con nuestro objetivo.

Palabras Clave: Visión Artificial, Redes Neuronales, Reconocimiento de Patrones, Reconocimiento de Mangos de Calidad.

\section{Abstract}

This article willtake as quality reference the ideal form of mangoes. The process is that, first we select a group of mangoes export that meet the quality requirements, then using a digital camera captures the image of each mango and by a image processing software, each mango is associated with a image containing only the figure of the edge of the mango mentioned. With these images, we proceed with the training of neural networks to find one that is able to recognize correctly all elements of the sample. Then we proced with the recognition tests 
using others mangoes that can have or not an ideal form. If the neural network trained recognizes adequately the form of the mango, we can say we have achieved our goal.

Key Words: Artificial Vision, Artificial Neural Networks, Pattern Recognition, Mangoes Quality Recognition.

\section{INTRODUCCIÓN}

La visión artificial cada día tiene más aplicaciones, y muchas veces se ha logrado que sea más precisa y rápida que la visión humana.

En el proceso de selección de mangos de calidad, la forma que tiene el mango es considerada como muy importante. Actualmente, uno de los criterios de selección para establecer la calidad de los mangos de exportación es la forma de dicha fruta. Este proceso es realizado manualmente por un grupo de personas especializadas, pero, a pesar de que lo realizan con mucho cuidado, siempre existen márgenes de errores considerables, lo que afecta importantemente en el negocio.

En este artículo, mostramos un procedimiento en el cual, una computadora captura mediante una cámara digital la imagen de un mango y, luego de un procesamiento previo basado en redes neuronales, se puede conocer si es un mango con una forma ideal de calidad o no lo es.

\section{SELECCIÓN DE MANGOS EX- PORTABLES}

Solamente trabajaremos con dos tipos de mangos: Haden y Ataulfo. Consultando con personas expertas, seleccio- namos tres unidades de mangos con calidad de exportación por cada tipo de mango.

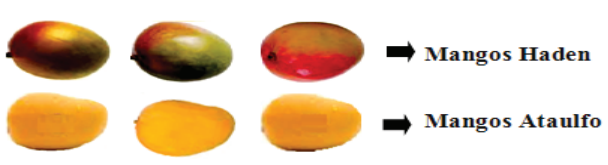

Figura 1. Mangos de calidad elegidos

\section{VISIÓN ARTIFICIAL PARA RECO- NOCIMIENTO DE UN MANGO}

La visión natural consiste en la capacidad de un ser vivo en captar una imagen a través del sentido de la vista e interpretar dicha imagen mediante un proceso mental (Spalton, Hitchings \& Hunter, 2006).

La visión artificial propuesta consiste en que una computadora mediante una cámara digital sea capaz de capturar la imagen de un mango y, mediante un procesamiento de imágenes, logre interpretarlo.

a. Se captura la imagen un mango mediante una cámara digital.

b. Se asigna una matriz con cuadrículas lo suficientemente pequeñas, para que contenga la imagen del mango. Para nuestro caso trabajamos con una matriz de dimensión 50 x 50 (Hilera, 1995).

c. Mediante un programa de procesamiento de imágenes, se obtiene el 
borde del mango el cual luego se representa usando las celdas de la matriz.

d. Se genera la matriz con datos binarios reemplazando las celdas vacías con 0s y las sombreadas con 1s.
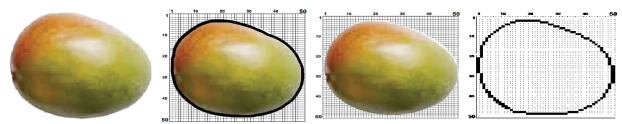

Figura 2. Proceso para la digitalización de la imagen de un mango

e. Luego se convierte la matriz binaria de 50 x 50 a un vector lineal binario de 2500 .

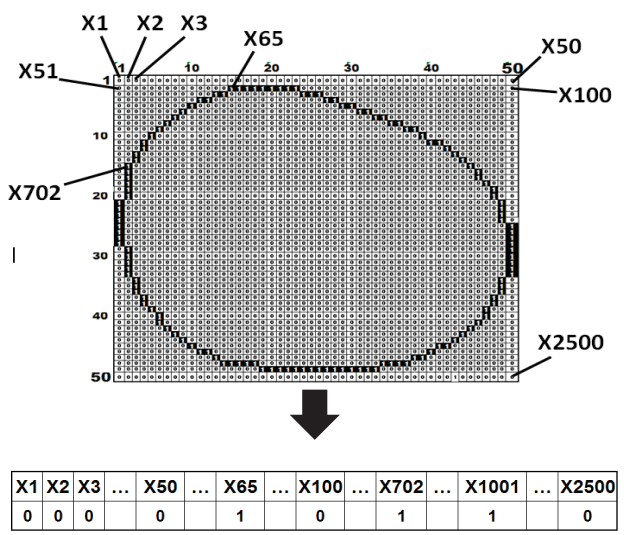

Figura 3. Conversión de la matriz binaria en un vector lineal

\section{RECONOCIMIENTO DE MANGOS DE CALIDAD USANDO REDES NEU- RONALES}

\subsection{Dependencia Funcional entre} los Datos de Entrada y Salida en una Red Neuronal

Para trabajar en el reconocimiento de patrones con redes neuronales, debemos preocuparnos primeramente de establecer el número de neuronas en la capa de entrada y el número de neuronas en la capa de salida. Por lo tanto, los datos de entrada estarán vinculados con las neuronas de entrada y los datos de salida con las neuronas de salida (Norgaard, 2000).

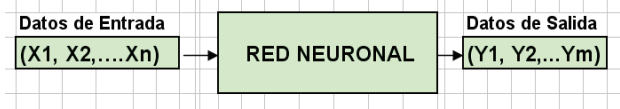

\section{Figura 4. Dependencia Funcional de los Datos de Entrada y Salida}

Estableciéndose entre ellos una dependencia funcional que la podemos llamar F, donde

DATOS DE SALIDA $=\mathrm{F}$ (DATOS DE ENTRADA) $[\mathrm{Y} 1, \mathrm{Y} 2, \ldots \mathrm{Ym}]=\mathrm{F}([\mathrm{X} 1, \mathrm{X} 2, \ldots \mathrm{Xn}])$

Como nuestro objetivo es demostrar que una red neuronal puede ser entrenada para reconocer los mangos de calidad, en el presente proyecto solamente trabajaremos con los mangos tipo Haden. Por lo tanto, la dependencia funcional de nuestra red neuronal será la siguiente:

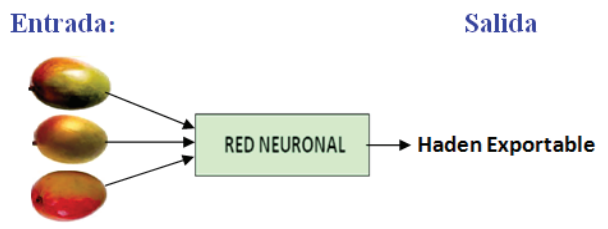

Figura 5. Dependencia funcional de los datos de entrada y salida (resumido)

\subsection{Arquitectura de la Red Neuronal para el Reconocimiento de Mangos}

Ahora estableceremos la arquitectura de la red neuronal. Es claro que el vector de entrada $X$, que almacena la imagen de un mango, consta de 2500 valores binarios (0s y 1s) y que el vector de salida Y solo consta de un valor binario. 
Por lo tanto, la red neuronal debe tener 2500 neuronas de entrada y una neurona de salida; las capas ocultas y el número de neuronas en ellas serán establecidas en el mismo proceso de entrenamiento (Fausett, 1993).

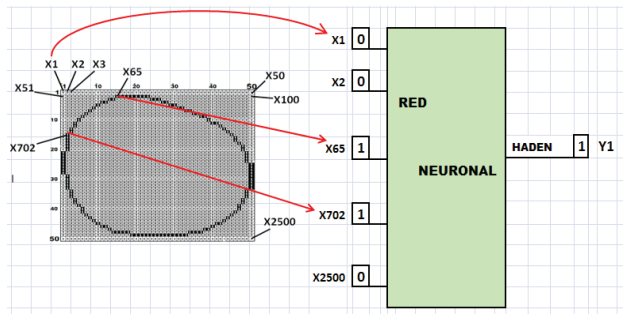

Figura 6. Arquitectura de la RN para el reconocimiento de mangos

\subsection{Generar la Base de Conocimien- to para el Entrenamiento de la Red Neuronal}

La base de conocimientos está conformada por la información que posee el experto humano; en este caso, estará conformada por la muestra de los mangos y su respectiva identificación de que si es exportable o no lo es.

\begin{tabular}{|l|c|}
\hline MANGO & CALIDAD \\
\hline & Haden Exportable \\
\hline & Haden Exportable \\
\hline & Haden Exportable \\
\hline
\end{tabular}

\section{Figura 7. Información real sobre los mangos}

Esta información debe ser almacenada en la computadora para usarlo en el entrenamiento de la red neuronal (Bryan, 2008) (Christopher, 2007).

Utilizando el concepto de visión artificial para el reconocimiento de mangos y siguiendo los pasos especifica- dos en el numeral 3, transformamos cada imagen en un vector binario de tamaño 2500.
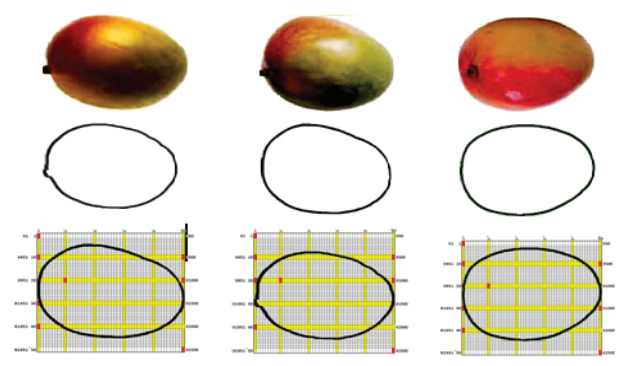

Figura 8. Generación de datos de entrada a partir de las imágenes de los mangos

Ya que a cada mango se le asignan 2500 valores binarios, por cuestiones de espacio, solamente mostraremos 10 valores para cada uno de ellos, que corresponden a los valores de las celdas resaltadas en rojo. Dichos valores son mostrados en la siguiente tabla.

Por lo tanto, la base de conocimientos, para el entrenamiento de nuestra res neuronal, será la siguiente.

\begin{tabular}{|c|c|c|c|c|c|c|c|c|c|c|c|c|}
\hline & & $\mathrm{x} 1$ & $x 451$ & X500 & X951 X & $\mathrm{X} 960 \mathrm{X1}$ & $\mathrm{x} 1000 \mathrm{x}$ & $\mathrm{x} 145$ & $51 \mathrm{X} 15$ & $500 \mathrm{x}$ & $\mathrm{x} 1951$ & $\mathrm{X} 2500$ \\
\hline \multicolumn{2}{|c|}{ Mango1 --: } & 0 & 0 & 0 & 1 & 0 & 0 & 0 & & 1 & 0 & 0 \\
\hline \multicolumn{2}{|c|}{ Mango2 -> } & 0 & 0 & 0 & 0 & 0 & 0 & 1 & & 1 & 0 & 0 \\
\hline \multicolumn{2}{|c|}{ Mango 3 ->> } & 0 & 0 & 0 & 1 & 0 & 1 & 0 & & 0 & 0 & 0 \\
\hline \multicolumn{13}{|c|}{ BASE DE CONOCIMIENTO PARA EL ENTRENAMIENTO DE LA RED NEURONAL } \\
\hline \multicolumn{12}{|c|}{ Datos de Entrada } & Salida \\
\hline $\mathrm{x} 1$ & $\mathrm{x} 451$ & $x 500$ & X951 & $x 960$ & $\mathrm{x} 1000$ & 01451 & $51 \times 150$ & & 1951 & $\times 250$ & & Y \\
\hline 0 & 0 & 0 & 1 & 0 & 0 & 0 & 1 & & 0 & 0 & & 1 \\
\hline 0 & 0 & 0 & 0 & 0 & 0 & 1 & 1 & & 0 & 0 & & 1 \\
\hline 0 & 0 & 0 & 1 & 0 & 1 & 0 & 0 & & 0 & 0 & & 1 \\
\hline
\end{tabular}

Figura 9. Base de conocimiento generada a partir de las Imágenes de los mangos

Los datos de este cuadro se guardan luego en un archivo tipo texto, digamos MUESTRAENT.TXT. Este ar- 
chivo será usado con el software de entrenamiento.

De ese modo, ahora ya tenemos nuestra base de conocimiento que contiene la información sobre los modelos ideales de los mangos HADEN de exportación.

\subsection{Entrenamiento de la Red Neu- ronal}

A continuación, mostraremos paso a paso el procedimiento de entrenamiento de una red neuronal; para ello utilizaremos nuestra base de conocimiento almacenada en MUESTRAENT.TXT y el software NEURONTRAINPATTERN.M, el cual ha sido creado en base al algoritmo (Teodoridis \& Koutroumbas, 2006).

\section{a. Primer Entrenamiento}

En el entorno del MATLAB, ejecutamos el programa NEURONTRAINPATTERN. M e ingresamos los parámetros requeridos.

Después del proceso, el software nos proporciona la imagen mostrada, donde se puede apreciar el seguimiento del proceso de aprendizaje de la RNA.

\begin{tabular}{ll|}
\hline $\begin{array}{l}\text { Archivo con data: } \\
\text { entrenamiento }\end{array}$ & MUESTRAENT.TXT $\rightarrow$ Base de \\
Sinapsis: Nueva $[0]$ & Se leerán desde un archivo [1]: $\mathbf{0}$ \\
Introducir neuronas en capa intermedia: & $\mathbf{1 0 0}$ \\
Introducir ratio de aprendizaje: & $\mathbf{0 . 0 5}$ \\
Introducir el valor máximo del error (\%): & $\mathbf{1 0}$ \\
Introducir el máximo etapas de aprendizaje: 200 \\
$\begin{array}{ll}\text { Archivo para resultado del entrenamiento: } & \text { ENTRENA- } \\
\text { MIENTO1.TXT }\end{array}$ \\
\hline
\end{tabular}

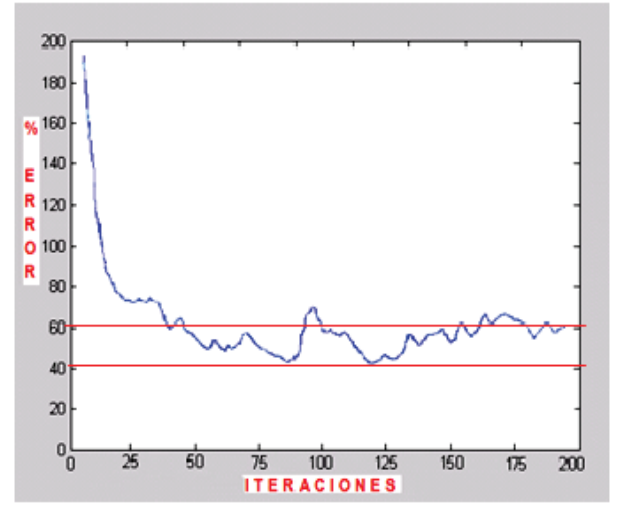

Figura 10. Ingreso de parámetros y Curva de un aprendizaje inadecuado

El gráfico muestra que el proceso de entrenamiento no ha sido satisfactorio, ya que se deseaba alcanzar un margen de error del $10 \%$ en un total máximo de 200 iteraciones, pero luego de las 200 iteraciones solo ha llegado al $60.03 \%$ del margen de error. Así mismo, se puede apreciar que aproximadamente en iteración 123 llegó al 40\% de margen error, aunque con un aprendizaje bastante irregular. En las iteraciones subsiguientes, en lugar de seguir disminuyendo dicho margen de error, empezó a crecer, también de modo irregular; por lo tanto, este entrenamiento se considera como no exitoso.

\section{b. Entrenamiento Óptimo}

De manera similar, realizamos un total de 33 pruebas de entrenamiento, buscando obtener el entrenamiento más óptimo, cambiando los parámetros de entrada del programa, hasta que en el entrenamiento número 21 , con los parámetros que a continuación mostramos, llegamos a un óptimo entrenamiento. 
Con estos nuevos parámetros, al finalizar el proceso de entrenamiento, el software nos proporciona la siguiente imagen, donde se puede apreciar una adecuada evolución del proceso de aprendizaje de la RNA:

\begin{tabular}{ll|}
\hline $\begin{array}{l}\text { Archivo con data: } \\
\text { entrenamiento }\end{array}$ & MUESTRAENT.TXT $\rightarrow$ Base de \\
Sinapsis: Nueva [0] & Se leerán desde un archivo [1]: $\mathbf{0}$ \\
Introducir neuronas en capa intermedia: & $\mathbf{4 0 0}$ \\
Introducir ratio de aprendizaje: & $\mathbf{0 . 0 2}$ \\
Introducir el valor máximo del error (\%): & $\mathbf{5}$ \\
$\begin{array}{l}\text { Introducir el máximo etapas de aprendizaje: } 200 \\
\text { Archivo para resultado del entrenamiento: }\end{array}$ & ENTRENA- \\
MIENTO-OK.TXT
\end{tabular}

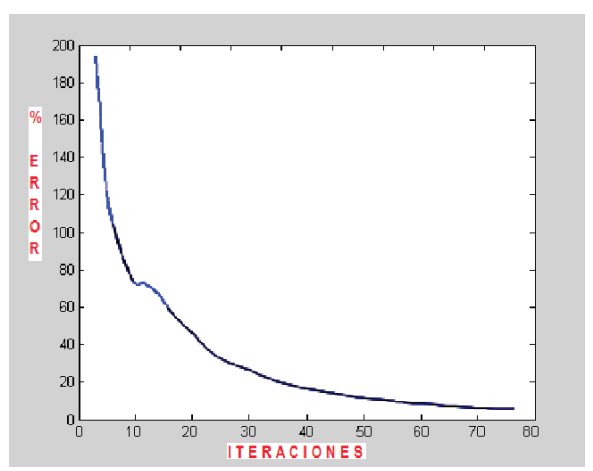

Figura 11. Ingreso de parámetros óptimos de entrenamiento y Curva de un aprendizaje óptimo

Como se puede apreciar, esta vez hemos alcanzado el $4.96 \%$ de margen de error en tan solo 78 iteraciones. Ahora tenemos una RNA bien entrenada para el reconocimiento de calidad de los mangos Haden exportables.

Debemos tener presente que el entrenamiento concluye guardando en un archivo tipo texto todos los pesos sinápticos de la red neuronal, como podemos apreciarlo en la última línea del proceso de entrenamiento óptimo; lo hemos guardado en el archivo ENTRENAMIENTO-OK.TXT. Este archivo será utilizado cuando se realice el PROCESO DE RECONOCIMIENTO de un mango de calidad.

\subsection{Reconocimiento Mangos con Ca- lidad de Exportación}

Recordemos que nuestros mangos tipo Haden para el entrenamiento, tal como lo presentamos en el numeral 2, fueron los siguientes:
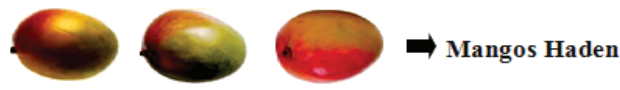

Figura 12. Imágenes de mangos para el entrenamiento de la red neuronal

Ahora, utilizaremos la red neuronal entrenada para realizar el proceso de reconocimiento de mangos con calidad de exportación; para ello, tomaremos una nueva muestra con un mango con calidad de exportación y dos mangos que no cumplen con dichas características.
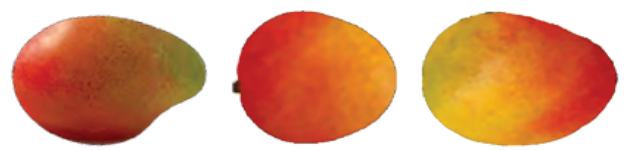

Figura 13. Imágenes de mangos para ser reconocidos por la red neuronal

Como se desea que la red neuronal determine si los mangos, son o no de calidad exportable, inicialmente diremos que esta característica es desconocida y que, precisamente, la red neuronal se encargará de establecerla. 


\begin{tabular}{|l|l|}
\hline MANGO & CALIDAD \\
\hline & Desconocida \\
\hline & Desconocida \\
\hline & Desconocida \\
\hline
\end{tabular}

Figura 14. Imágenes de mangos para el proceso de reconocimiento

Con esta información, de manera similar al proceso de entrenamiento, usando el proceso de visión artificial, generamos los datos de entrada para cada mango.

\begin{tabular}{|c|c|c|c|c|c|c|c|c|c|c|}
\hline \multicolumn{10}{|c|}{ DATOS BINARIOS DE LOS MANGOS PARA EL RECONOMIENTO DE CALIDAD } \\
\hline X1 & X451 & X500 & X951 & X960 & X1000 & X1451 & X1500 & X1951 & X2500 & Y Salida \\
\hline 0 & 0 & 0 & 0 & 0 & 0 & 1 & 1 & 0 & 0 & 0 \\
\hline 0 & 0 & 0 & 0 & 0 & 0 & 0 & 1 & 0 & 0 & 0 \\
\hline 0 & 0 & 0 & 1 & 0 & 0 & 1 & 0 & 0 & 0 & 0 \\
\hline
\end{tabular}

Figura 15. Datos binarios de los mangos a ser reconocidos por la red neuronal

Debemos tener presente que los valores correspondientes a la variable de salida serán cero, debido a que los mangos respectivos son de calidad desconocida.

Dichos datos binarios que representan a los mangos a ser reconocidos, lo guardamos en un archivo tipo texto MUESTRAREC.TXT y, luego, procedemos a usar el NUROTRAINPATTREN.M para reconocer la característica de calidad de dichos mangos.

\section{a. Proceso de Reconocimiento Uti-} lizando la Red Neuronal Entrenada.

En el entorno del MATLAB, ejecutamos el programa NEURONTRAINPATTERN. M e ingresamos los parámetros requeridos.

El proceso de reconocimiento es muy similar al entrenamiento. Prime- ro, en el entorno MATLAB, ejecutamos el programa NEURONTRAINPATTERN e ingresamos los parámetros requeridos, según se muestra a continuación. Archivo con data: MUESTRAREC.TXT $\rightarrow$ Base de
Reconocimiento
Archivo de entrenamiento: ENTRENAMIENTOOK.TXT
Archivo de salida: SALIDA.TXT

Figura 16. Proceso de reconocimiento de patrones

Al finalizar el proceso de reconocimiento, se genera un archivo de salida, en este caso lo llamamos SALIDA. TXT, el cual al abrirlo nos muestra la siguiente información.

$$
\begin{aligned}
& 0.341 \\
& 0.469 \\
& 0.813
\end{aligned}
$$

Figura 17. Información de salida de la red neuronal

\section{b. Interpretación de los Datos de Salida}

Los valores tomados del archivo de salida pasados al Excel se aprecian de la siguiente manera:

\begin{tabular}{|l|}
\hline$Y$ \\
\hline 0 \\
\hline 0 \\
\hline 1 \\
\hline
\end{tabular}

\begin{tabular}{|c|}
\hline $\mathrm{Y}$ \\
\hline 0.341 \\
\hline 0.469 \\
\hline 0.813 \\
\hline
\end{tabular}

Figura 18. Datos de salida y con valores reales y valores redondeados

Apreciando los valores, podemos mencionar que:

- $\mathrm{Y}=0.341$ quiere decir que el primer mango tiene solamente el $34.1 \%$ de las exigencias de calidad para un mango de exportación. En otras palabras, la red 
neuronal lo reconoce como un mango defectuoso o no exportable

- $\mathrm{Y}=0.469$ quiere decir que el segundo mango tiene solamente el $46.9 \%$ de las exigencias de calidad para un mango de exportación. En otras palabras, la red neuronal lo reconoce como un mango defectuoso o no exportable.

- $\mathrm{Y}=0.813$ quiere decir que el tercer mango tiene un $81.3 \%$ de las exigencias de calidad para un mango de exportación. En otras palabras, la red neuronal lo reconoce como un mango exportable.

- Así mismo, apreciando los valores redondeados, podemos afirmar que el 1 significa que el mango respectivo ha sido reconocido por nuestra la red neuronal como un mango exportable y el 0 significa el mango ha sido reconocido como no exportable.

\section{CONCLUSIONES}

En el presente artículo, hemos presentado un modelo simple de visión artificial, el cual puede ser aplicado a otros campos, tales como la medicina, biología u otros.

Este resultado no pudo haber sido posible sin un software que nos permita el procesamiento de imágenes, el mismo que nos ha permitido obtener los bordes de las imágenes de cada mango.

\section{REFERENCIAS BIBLIOGRÁFICAS}

Spalton, J. Hitchings, Roger A, Hunter, Paul (2006). "Atlas de oftalmología clínica", Elsevier España

Hilera, J. R. (1995). "Redes Neuronales Artificiales. Fundamentos Modelos y Aplicaciones", RA-MA.

Norgaard, M. (2000). "Neural Networks for Modelling and Control of Dynamic Systems”, Spinger-Verlag L. Limited (2000).

Fausett, Laurene (1993). "Fundamentals of Neural Networks: Architectures, Algorithms And Applications", Prentice Hall.

Ripley, Brian D. (2008). "Pattern Recognition and Neural Networks", Cambrige University Press.

Bishop, Christopher M. (2007). "Pattern Recognition and Machine Learning", Springer.

Theodoridis, Sergios, Koutroumbas, Konstantinos (2006). "Pattern Recognition", Academic Press. 